K.A. van der Hucht, G. Koenigsberger \& P.R.J. Eenens, eds.

\title{
High-resolution spectroscopic study of the planetary nebula Abell 78
}

\author{
Selene Medina and Miriam Peña \\ Instituto de Astronomía, UNAM, \\ Apdo. 70-264, México, D.F., 04510, México
}

\begin{abstract}
High resolution optical spectra of the planetary nebula Abell 78 were gathered, covering the wavelength ranges $3500-6600 \AA$ and $3358-7361 \AA$, with a spectral resolution of $\sim 0.1 \AA$. Two different regions of Abell 78 are analyzed: the strongest knot near the central star which is thought to be a massloaded wind from the central star, and the outer, hydrogen-rich envelope. Physical parameters and chemical composition of both regions are estimated. The inner knot appears to be cooler and denser than the hydrogen-rich envelope. The chemical composition of the inner knot shows that there is almost no hydrogen near the central star and the emission is dominated by helium and processed material which has been dredged-up from the inner layers of the star. The outer, hydrogen-rich envelope appears to be deficient in heavy elements, mainly in oxygen, compared to the average abundances that are found among galactic planetary nebulae.
\end{abstract}

\section{Introduction}

Abell 78 is a member of a small but important class of planetary nebulae, which are characterized by hydrogen-poor, dusty ejecta. In these objects the hydrogenpoor material is surrounded by an outer envelope of normal composition, which indicates that a secondary ejection of highly processed material has occurred after the loss of the hydrogen envelope of the AGB progenitor.

Harrington et al. (1995) obtained spectroscopic observations of the central star with HST in the vicinity of the C IV $\lambda \lambda 1548.2,1550.8$ doublet and found a series of blue-shifted absorption features superposed on the broad P-Cygni profile, in the velocity range of 18 to $\sim 385 \mathrm{~km} \mathrm{~s}^{-1}$, giving the evidence of massloading in Abell 78.

The present work provides an estimate of the physical parameters and chemical composition of both the mass-loaded wind from the central star and the hydrogen-rich envelope.

\section{Observations}

Optical spectra of the strongest knot near the central star and the outer, hydrogen-rich envelope of Abell 78 were gathered with the ESPRESSO echelle-spectrograph using the 2.1-m telescope at San Pedro Mártir, México, covering the wavelength ranges $3500-6600 \AA$ and $3358-7361 \AA$, with a spectral resolution of $\sim 0.1 \AA$. The integration time was 15 minutes in all cases. 


\section{Discussion}

The physical parameters and chemical composition were estimated, assuming that photo-ionization is the only mechanism that produces the observed emission lines. This estimate gives values of $19100 \pm 1000 \mathrm{~K}$ and $3000 \pm 1000 \mathrm{~cm}^{-3}$ for electron temperature and density, respectively, for the inner knot; and $21400 \pm$ $2000 \mathrm{~K}$ and $1000 \pm 300 \mathrm{~cm}^{-3}$ for the outer, hydrogen-rich envelope.

The chemical composition of the inner knot shows, that there is almost no hydrogen near the central star and the emission is dominated by helium. There are traces of processed material such as oxygen and neon, which probably have been dredged-up from the inner layers of the star.

On the other hand, the hydrogen-rich envelope appears to be deficient in heavy elements, compared to the average abundances that are found among galactic planetary nebulae. For instance, the abundance by number of oxygen is about 5 times smaller than the average value reported by Kingsburgh \& Barlow (1994). There are two possible explanations: firstly, the progenitor star was probably born in an intrinsically metal-deficient medium or, secondly, this object is now passing through a particular stage of its evolution in which the oxygen appears to be diminished.

The isophotal diagram of the [OIII] $\lambda 5007 \AA$ line in the inner knot shows a structure that appears to arise from a non-spherical movement of the gas, from a jet-like ejection. The velocity structure of this line has a velocity of $\sim 100$ $\mathrm{km} \mathrm{s}^{-1}$ relative to the central star.

Nebular emission of [OI] $\lambda 6300 \AA$ and $\mathrm{H} \alpha$ was detected in both regions. Their isophotal diagrams show almost the same feature, which may be due to the fact that both lines come from the very same region of the nebula and, therefore, from a region with the same kinematics.

Acknowledgments. The authors are grateful to G. Stasińska for supplying the ABELTON program, which calculated the physical parameters and ionic abundances from the observed data. This work was partially supported by DGAP A/UNAM (grant IN-109696).

\section{References}

Harrington, J.P., Borkowski, K.J., Tsvetanov, Z. 1995, ApJ 439, 264

Kingsburgh, R.L., Barlow, M.J. 1994, MNRAS 271,257 